\title{
Erratum: Gruber, E.S.; et al. The Oncogene AF1Q is Associated with WNT and STAT Signaling and Offers a Novel Independent Prognostic Marker in Patients with Resectable Esophageal Cancer. Cells 2019, 8, 1357
}

\author{
Elisabeth S. Gruber ${ }^{1,2}{ }^{\oplus}$, Georg Oberhuber ${ }^{3,4}$, Peter Birner ${ }^{3}$, Michaela Schlederer ${ }^{3}$, \\ Michael Kenn ${ }^{5}$, Wolfgang Schreiner ${ }^{5}{ }^{-}$, Gerd Jomrich ${ }^{1,2}{ }^{-}$, Sebastian F. Schoppmann ${ }^{1,2}$, \\ Michael Gnant ${ }^{2}$, William Tse ${ }^{6,7, *}$ and Lukas Kenner ${ }^{3,8,9,10, * \mathbb{D}}$ \\ 1 Division of General Surgery, Department of Surgery, Medical University of Vienna, 1090 Vienna, Austria; \\ elisabeth.s.gruber@meduniwien.ac.at (E.S.G.); gerd.jomrich@meduniwien.ac.at (G.J.); \\ sebastian.schoppmann@meduniwien.ac.at (S.F.S.) \\ 2 Comprehensive Cancer Center, Medical University of Vienna, 1090 Vienna, Austria; \\ michael.gnant@meduniwien.ac.at \\ 3 Department of Experimental and Translational Pathology, Institute of Pathology, \\ Medical University of Vienna, 1090 Vienna, Austria; georg.oberhuber@patho.at (G.O.); \\ peter.birner@meduniwien.ac.at (P.B.); michaela.schlederer@meduniwien.ac.at (M.S.) \\ 4 PIZ-Patho im Zentrum GmbH, 3100 St. Poelten, Austria \\ 5 Section of Biosimulation and Bioinformatics, Center for Medical Statistics, Informatics and Intelligent \\ Systems (CeMSIIS), Medical University of Vienna, 1090 Vienna, Austria; \\ michael.kenn@meduniwien.ac.at (M.K.); wolfgang.schreiner@meduniwien.ac.at (W.S.) \\ 6 James Graham Brown Cancer Center, University of Louisville School of Medicine, Louisville, KY 40202, USA \\ 7 Division of Blood and Bone Marrow Transplantation, Department of Medicine, \\ University of Louisville School of Medicine, Louisville, KY 40202, USA \\ 8 Christian Doppler Laboratory for Applied Metabolomics (CDL-AM), Medical University of Vienna, \\ 1090 Vienna, Austria \\ 9 Institute of Laboratory Animal Pathology, University of Veterinary Medicine Vienna, 1210 Vienna, Austria \\ 10 CBmed Core Lab 2, Medical University of Vienna, 1090 Vienna, Austria \\ * Correspondence: wtse@metroHealth.org (W.T.); lukas.kenner@meduniwien.ac.at (L.K.); \\ Tel.: +1-216-778-3845 (W.T.); +43-40400-51760 (L.K.)
}

The authors wish to make the following change to their paper [1]: The Affiliations section is incorrect in the published paper with regards to the affiliation numbers of the authors. In addition, the e-mail address and phone number of the corresponding author has been updated.

The original version is:

Elisabeth S. Gruber ${ }^{1}$, Georg Oberhuber ${ }^{2,3}$, Peter Birner ${ }^{2}$, Michaela Schlederer ${ }^{2}$, Michael Kenn ${ }^{4}$, Wolfgang Schreiner ${ }^{4}$, Gerd Jomrich ${ }^{1}$, Sebastian F. Schoppmann ${ }^{1}$, Michael Gnant ${ }^{1}$, William Tse ${ }^{5,6, *}$ and Lukas Kenner $2,7,8,9, *$

1 Division of General Surgery, Department of Surgery, Comprehensive Cancer Center, Medical University of Vienna, 1090 Vienna, Austria; elisabeth.s.gruber@meduniwien.ac.at (E.S.G.); gerd.jomrich@meduniwien.ac.at (G.J.); sebastian.schoppmann@meduniwien.ac.at (S.F.S.); michael.gnant@meduniwien.ac.at (M.G.)

2 Department of Experimental and Translational Pathology, Institute of Pathology, Medical University of Vienna, 1090 Vienna, Austria; georg.oberhuber@patho.at (G.O.); peter.birner@meduniwien.ac.at (P.B.); michaela.schlederer@meduniwien.ac.at (M.S.) 
${ }^{3}$ PIZ-patho im zentrum GmbH, 3100 St. Poelten, Austria

${ }^{4}$ Section of Biosimulation and Bioinformatics, Center for Medical Statistics, Informatics and Intelligent Systems (CeMSIIS), Medical University of Vienna, 1090 Vienna, Austria; michael.kenn@meduniwien.ac.at (M.K.); wolfgang.schreiner@meduniwien.ac.at (W.S.)

${ }^{5}$ James Graham Brown Cancer Center, University of Louisville School of Medicine, Louisville, KY 40202, USA

${ }^{6}$ Division of Blood and Bone Marrow Transplantation, Department of Medicine, University of Louisville School of Medicine, Louisville, KY 40202, USA

${ }^{7}$ Christian Doppler Laboratory for Applied Metabolomics (CDL-AM), Medical University of Vienna, 1090 Vienna, Austria

8 Institute of Laboratory Animal Pathology, University of Veterinary Medicine Vienna, 1210 Vienna, Austria

${ }^{9}$ CBmed Core Lab 2, Medical University of Vienna, 1090 Vienna, Austria

* Correspondence: william.tse@louisville.edu (W.T.); lukas.kenner@meduniwien.ac.at (L.K.); Tel.: +1-502-562-4363 (W.T.); +43-1-40400-51760 (L.K.)

This should be replaced with:

Elisabeth S. Gruber ${ }^{1,2}$, Georg Oberhuber ${ }^{3,4}{ }^{4}$ Peter Birner ${ }^{3}$, Michaela Schlederer ${ }^{3}$, Michael Kenn ${ }^{5}$, Wolfgang Schreiner ${ }^{5}$, Gerd Jomrich ${ }^{1,2}$, Sebastian F. Schoppmann ${ }^{1,2}$, Michael Gnant ${ }^{2}$, William Tse ${ }^{6,7, *}$ and Lukas Kenner ${ }^{3,8,9,10, *}$

1 Division of General Surgery, Department of Surgery, Medical University of Vienna, 1090 Vienna, Austria; elisabeth.s.gruber@meduniwien.ac.at (E.S.G.); gerd.jomrich@meduniwien.ac.at (G.J.); sebastian.schoppmann@meduniwien.ac.at (S.F.S.)

2 Comprehensive Cancer Center, Medical University of Vienna, 1090 Vienna, Austria; michael.gnant@meduniwien.ac.at

${ }^{3}$ Department of Experimental and Translational Pathology, Institute of Pathology, Medical University of Vienna, 1090 Vienna, Austria; georg.oberhuber@patho.at (G.O.); peter.birner@meduniwien.ac.at (P.B.); michaela.schlederer@meduniwien.ac.at (M.S.)

${ }^{4}$ PIZ—Patho im Zentrum GmbH, 3100 St. Poelten, Austria

${ }^{5}$ Section of Biosimulation and Bioinformatics, Center for Medical Statistics, Informatics and Intelligent Systems (CeMSIIS), Medical University of Vienna, 1090 Vienna, Austria; michael.kenn@meduniwien.ac.at (M.K.); wolfgang.schreiner@meduniwien.ac.at (W.S.)

6 James Graham Brown Cancer Center, University of Louisville School of Medicine, Louisville, KY 40202, USA

${ }^{7}$ Division of Blood and Bone Marrow Transplantation, Department of Medicine, University of Louisville School of Medicine, Louisville, KY 40202, USA

${ }^{8}$ Christian Doppler Laboratory for Applied Metabolomics (CDL-AM), Medical University of Vienna, 1090 Vienna, Austria; lukas.kenner@meduniwien.ac.at (L.K.)

9 Institute of Laboratory Animal Pathology, University of Veterinary Medicine Vienna, 1210 Vienna, Austria

${ }^{10}$ CBmed Core Lab 2, Medical University of Vienna, 1090 Vienna, Austria

* Correspondence: wtse@metroHealth.org (W.T.); lukas.kenner@meduniwien.ac.at (L.K.); Tel.: +1-216-778-3845 (W.T.); +43-40400-51760 (L.K.)

We apologize for any inconvenience caused to the readers by this mistake. The manuscript will be updated and the original will remain online on the article webpage. 


\section{Reference}

1. Gruber, E.S.; Oberhuber, G.; Birner, P.; Schlederer, M.; Kenn, M.; Schreiner, W.; Jomrich, G.; Schoppmann, S.F.; Gnant, M.; Tse, W.; et al. The Oncogene AF1Q is Associated with WNT and STAT Signaling and Offers a Novel Independent Prognostic Marker in Patients with Resectable Esophageal Cancer. Cells 2019, 8, 1357. [CrossRef] [PubMed]

Publisher's Note: MDPI stays neutral with regard to jurisdictional claims in published maps and institutional affiliations.

(C) 2020 by the authors. Licensee MDPI, Basel, Switzerland. This article is an open access article distributed under the terms and conditions of the Creative Commons Attribution (CC BY) license (http://creativecommons.org/licenses/by/4.0/). 